\title{
Assessing migraine patients with multifocal pupillographic objective perimetry
}

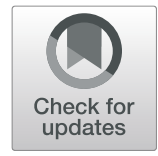

\author{
Eman N. Ali, ${ }^{1,2}$, Corinne F. Carle ${ }^{1}$, Christian J. Lueck ${ }^{3,4}\left(\mathbb{D}\right.$, Maria Kolic $^{1}$ (D) and Ted Maddess ${ }^{1 *}(\mathbb{D}$
}

\begin{abstract}
Background: To establish the effects of stimulating intrinsically-photosensitive retinal ganglion cells (ipRGCs) on migraine severity, and to determine if migraine produces objectively-measured visual field defects.

Methods: A randomized, open labelled, crossover study tested migraineurs and normal controls using multifocal pupillographic objective perimetry (mfPOP) with 44 test-regions/eye. A slow blue protocol (BP) stimulated ipRGCs, and a fast yellow protocol (YP) stimulated luminance channels. Migraine diaries assessed migraine severity. Perregion responses were analyzed according to response amplitude and time-to-peak.

Results: Thirty-eight migraineurs ( $42.0 \pm 16.5$ years, 23 females) and 24 normal controls ( $39.2 \pm 15.2$ years, 14 females) were tested. The proportion of subjects developing a migraine did not differ after either protocol, either during the 1st day (odds ratio 1.0; 95\% confidence interval 0.2-4.4, $p=0.48$ ) or during the first 3 days after testing (odds ratio 0.8; 95\% confidence interval 0.3-2.1, $p=0.68$ ). Migraine days/week did not increase following testing with either protocol in comparison to the baseline week (1.4 \pm 1.6 pre-testing (mean \pm SD), $1.3 \pm 1.4$ post-BP, and $1.3 \pm 1.2$ post-YP; $p=0.96)$, neither did other measures of severity. Migraine occurring up to 2 weeks before testing significantly lowered amplitudes, $-0.64 \pm 0.14 \mathrm{~dB}$ (mean $\pm \mathrm{SE}$ ), while triptan use increased amplitudes by $0.45 \pm 0.10$ $\mathrm{dB}$, both at $p<0.001$.
\end{abstract}

Conclusions: Stimulating ipRGCs did not affect migraine occurrence or severity. Pupillary response characteristics were influenced by the occurrence of a recent migraine attack and a history of triptan use.

Keywords: Migraine, Trigeminovascular pathway, Melanopsin, Multifocal pupillography, Photosensitivity

\section{Background}

Migraine is thought to be associated with cortical spreading depression (CSD) which consists of a propagated wave of profound depression in cerebral cortical neural activity preceded by transient neuronal activation. CSD is believed to underlie migraine aura and to be a trigger for the headache pain [1]. Light is a wellrecognized trigger of migraine attacks [2], and photophobia is a core feature of the condition but the mechanism is not fully understood. Noseda et al. [3] have described a retino-thalamic pathway involving the

\footnotetext{
* Correspondence: ted.maddess@anu.edu.au

${ }^{1}$ Eccles Institute of Neuroscience, the John Curtin School of Medical Research, Australian National University, Acton, ACT, Australia

Full list of author information is available at the end of the article
}

intrinsically-photosensitive retinal gangion cells (ipRGCs), which may be responsible for photophobia [4, 5]. While the ipRGCs receive input from rods and cones they also contain the photosensitive pigment, melanopsin, and so are themselves sensitive to blue light in bright conditions [6]. They relay their responses to cells in the posterior thalamus, among other targets. The thalamic target cells also receive input from the trigeminovascular pathway, which is believed to carry the pain signals arising from the dura mater during migraine attacks [3], and send their output to the somatosensory cortex. Intense blue stimuli of up to $1 \mathrm{~s}$ are generally required to activate the melanopsin response of ipRGCs [6-8], and their slow responses govern the steady-state size of the pupil [9]. Human studies of the contribution

C C The Author(s). 2021 Open Access This article is licensed under a Creative Commons Attribution 4.0 International License, which permits use, sharing, adaptation, distribution and reproduction in any medium or format, as long as you give appropriate credit to the original author(s) and the source, provide a link to the Creative Commons licence, and indicate if changes were made. The images or other third party material in this article are included in the article's Creative Commons licence, unless indicated otherwise in a credit line to the material. If material is not included in the article's Creative Commons licence and your intended use is not permitted by statutory regulation or exceeds the permitted use, you will need to obtain permission directly from the copyright holder. To view a copy of this licence, visit http://creativecommons.org/licenses/by/4.0/ The Creative Commons Public Domain Dedication waiver (http://creativecommons.org/publicdomain/zero/1.0/) applies to the data made available in this article, unless otherwise stated in a credit line to the data. 
of melanopsin to responses of the pupils have found stimuli of 1 to several seconds to be optimal [10-12]. A previous study conducted by Main et al. [13] observed that migraine patients found both short (blue) and long (red) wavelengths of light significantly more uncomfortable between attacks compared to normal controls and subjects with tension-type headache. However, those results were based on purely subjective measures and, overall, a method for objectively assessing ipRGC function in migraineurs would be useful.

Multifocal Pupillographic Objective Perimetry (mfPOP) is a developing diagnostic technique that assesses visual function objectively using pupillary responses. As is common in recent mfPOP studies, mfPOP protocols used here [14] assessed 44 locations in the visual fields of both eyes concurrently. By testing both eyes with 88 sets of independent stimuli, and recording the response of both pupils, the device can distinguish localized afferent and efferent defects $[15,16]$ within a few minutes. Alterations in pupillary responses have previously been described clinically in migraine patients: prolonged mydriasis has been reported during migraine attacks, sometimes persisting for up to 3 months after an attack, suggesting dysfunction of the parasympathetic supply of the pupils $[17,18]$. However, conventional pupillometry has so far failed to confirm these findings [17]. Localized visual field defects have been reported in migraine using various forms of perimetry [19-22], but this is not a consistent finding [23]. Localized visual field changes in terms of both sensitivity and response delay have been reported using $\mathrm{mfPOP}$ in diabetic retinopathy [15], macular degeneration [24], glaucoma [14], multiple sclerosis [25], and concussion [26]. In simultaneous mfPOP and multifocal visual evoked potential (mfVEP) recordings higher-sensitivity is observed in extra-striate cortex in association with earlystage retinal disease [27], and attentional effects can also be quantified by mfPOP [28]. The ability to detect cortically-mediated effects suggested that mfPOP could be used to investigate migraineurs. Most mfPOP studies have used transiently-presented yellow stimuli designed to minimize the effects of lens brunescence and attention [28], although we have reported studies using red/ green equiluminant mfPOP stimuli [29]. We have recently reported on blue mfPOP stimuli that strongly favor ipRGCs [14], which are 30 times longer in duration that traditionally transient mfPOP stimuli. Given the possibility that ipRGCs are involved in the pathogenesis of migraine [3] we decided to incorporate these longduration blue stimuli in a study of migraine.

This study had three aims: first, to determine whether testing migraine patients with ipRGC-biased blue mfPOP stimuli would provoke/exacerbate symptoms when compared to the standard transient yellow stimuli designed to drive dynamic luminance-sensitive channels. Second, to determine the ability of either stimulus to detect pupillary abnormalities in the days and weeks following a migraine attack. Third, to detect any change of sensitivity or delay in the per-region responses to visual field stimulation in migraineurs compared to normal controls.

\section{Methods}

\section{Study design and subjects}

A randomized, controlled, open-label, crossover, singlesite study was undertaken over 1 year (Fig. 1). Subjects with migraine were recruited from staff and students at The Australian National University and via local neurologists at The Canberra Hospital in Canberra, Australia. Informed, written consent was obtained from all subjects. The study conformed to the Declaration of Helsinki guidelines and was approved by both the $\mathrm{Hu}$ man Research Ethics Committee of the Australian National University (2012/278) and the ACT Health Human Research Ethics Committee (ETH.3.12.064).

Inclusion criteria comprised: 1) age above 18 years, 2) a clear diagnosis of migraine with or without aura according to International Headache Society criteria [30], 3) corrected visual acuity in both eyes better than $6 / 12$. Exclusion criteria included: 1) a history of other visual or neurological disturbance that might affect visual assessment, 2) a history of epilepsy, 3) colour blindness, 4) pregnancy, or 5) medication that could affect pupillary responses including miotics, antidepressants and some common decongestants [31], 6) migraine headache occurring within the 24-h period before testing. The control group consisted of age- and sex-matched participants who underwent a standard eye examine by MK including slit-lamp and acuity, and had the same medical history exam as the migraineurs by EA.

Sample size calculation suggested that a total of $22 \mathrm{mi}-$ graine subjects and 22 controls would be needed in order to detect an effect size of $40 \%$ increase in migraine headache or aura occurrence after testing with the mfPOP device. The power was set at $80 \%$ using a twosided t-test at the level of $p=0.05$. This calculation was done using the sample size formula for proportions [32].

A screening session to establish eligibility was performed for each participant during which background information regarding age at migraine onset, typical triggers, pattern, frequency and duration of migraine, presence of photophobia, other headaches, time since the most recent headache attack, and medication use (therapeutic or preventative) was obtained. Medications were divided according to class into: triptans, opioids, or over-the-counter (OTC) medications (NSAIDs, aspirin and paracetamol). 


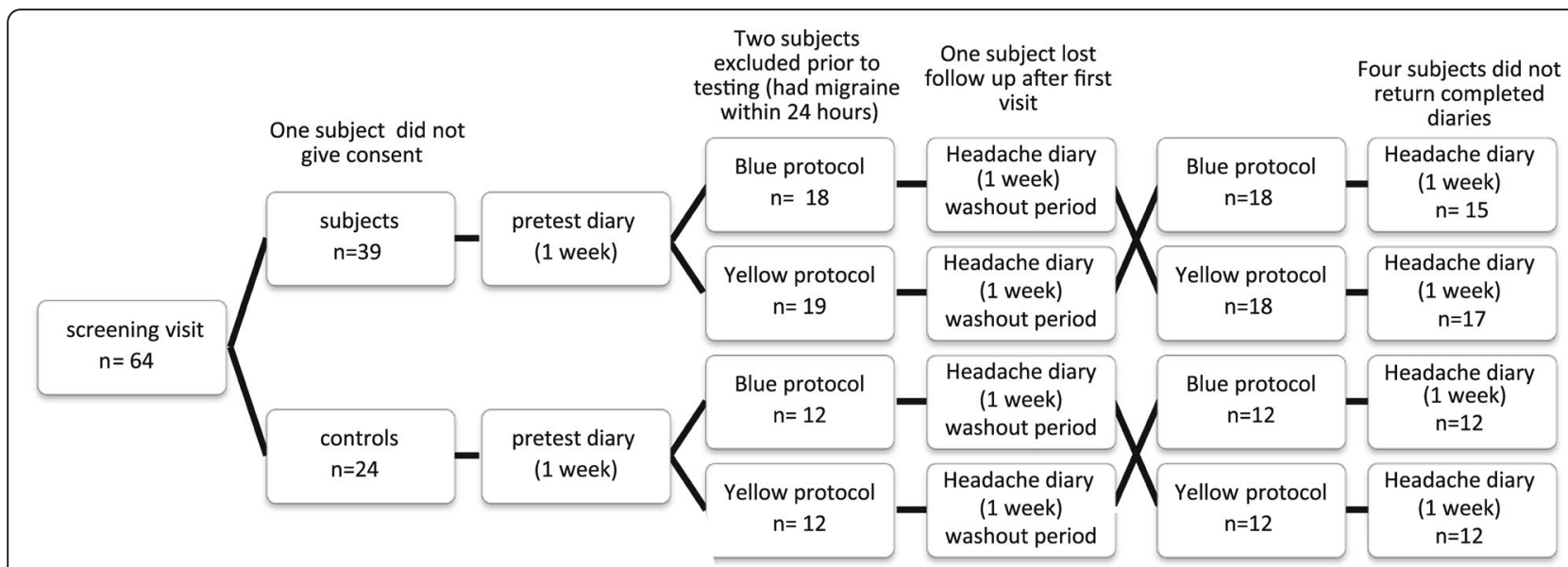

Fig. 1 Study cross-over design. One-week headache diaries were completed before testing, before the 1-week washout period, and after the second test involving a crossover between yellow and blue stimuli

Subjects and controls were asked to complete a baseline headache diary for 1 week. Participants were then randomized using Research Randomizer software [33] to undergo one of the two mfPOP testing protocols: transient-yellow or slow-blue (Fig. 1). The randomized crossover design minimized the influence of confounding covariates because each patient acted as their own control. After each test a second diary was completed for a week followed by a washout period of a week. Subjects then underwent the other mfPOP protocol after which they completed a third headache diary. For ethical reasons, the use of subjects' usual pain-relieving medications was permitted. This was felt to be likely to increase adherence to the study and also enabled evaluation of the effects of medication use before and after testing. All participants were advised not to smoke, drink caffeinated beverages, or consume alcohol for $6 \mathrm{~h}$ before mfPOP testing.

\section{Migraine diaries}

A validated migraine headache diary devised by The Diagnostic Headache Diary Study Group [34] was used on the three occasions described above. Parameters recorded included whether the subject experienced a migraine headache (yes/no), severity on a scale of $1-3(1=$ not bad, 2 = quite bad, 3 = very bad), duration (estimated from the time the first symptoms were noticed until the time the headache finally subsided), characteristics (throbbing or compressing /unilateral or bilateral), associated factors (presence of aura, photophobia, phonophobia, nausea, vomiting), precipitating and relieving factors, as well as medication consumption (including type, dosage and frequency).

\section{mfPOP assessment}

All subjects underwent mfPOP assessment using the FDA-cleared objectiveFIELD Analyser ${ }^{\ominus}$ (OFA) prototype
(Konan Medical USA, Irvine CA) (Fig. 2). Figure 2a shows a schematic of the device. Corrective lenses compensated for refractive errors. Trains of pseudo-randomly presented stimuli were presented at 44 possible locations/eye concurrently (Fig. 4b). The characteristics of the Yellow Protocol (YP) and the Blue Protocol (BP) are given in Table 1 . The same stimuli have been used previously in a study of glaucoma and more details are provided there [14]. Aside from data presented there, the data of [35, 36] indicate that at the background and stimulus intensities used here pupil responses to blue stimuli are substantially driven by melanopsin. The $1 \mathrm{~s}$ duration of the BP stimuli is also optimal for such responses [10].

The spatial layouts of YP and BP were identical: the locations where stimuli could occur comprised a dartboard like pattern consisting of 5 rings of stimuli extending to $\pm 30^{\circ}$ eccentricity of visual field (Fig. 2b). Figure $2 \mathrm{c}$ shows that over time the stimuli were presented in randomized clustered volleys [37]. The possible stimuli within a volley consisted of hemifield-sets of either the two rings defined by solid lines in Fig. $2 b$, or the 3 rings defined by dashed lines. Periodically, every $0.25 \mathrm{~s} /$ region for YP, and $0.5 \mathrm{~s} /$ region for $\mathrm{BP}$, a given hemifield/ ring set had the opportunity to be displayed, with $50 \%$ probability for any one stimulus location. This sequencing was designed to keep pupil sensitivity high and balanced over time [38]. When presented, individual stimuli remained on for different durations: $33 \mathrm{~ms}$ for YP, and $1000 \mathrm{~ms}$ for BP. Both protocols ran for $360 \mathrm{~s}$, presented in 9 segments of $40 \mathrm{~s}$ duration to allow rest breaks. This meant that, on average, the mean interval between stimuli experienced by a given visual field region was $4 \mathrm{~s}$ for YP, and $8 \mathrm{~s}$ for BP. For YP and BP this meant each region was tested 90 or 45 times respectively. Examples of the resulting averaged pupil responses from a single YP test are presented in Fig. 3. The 

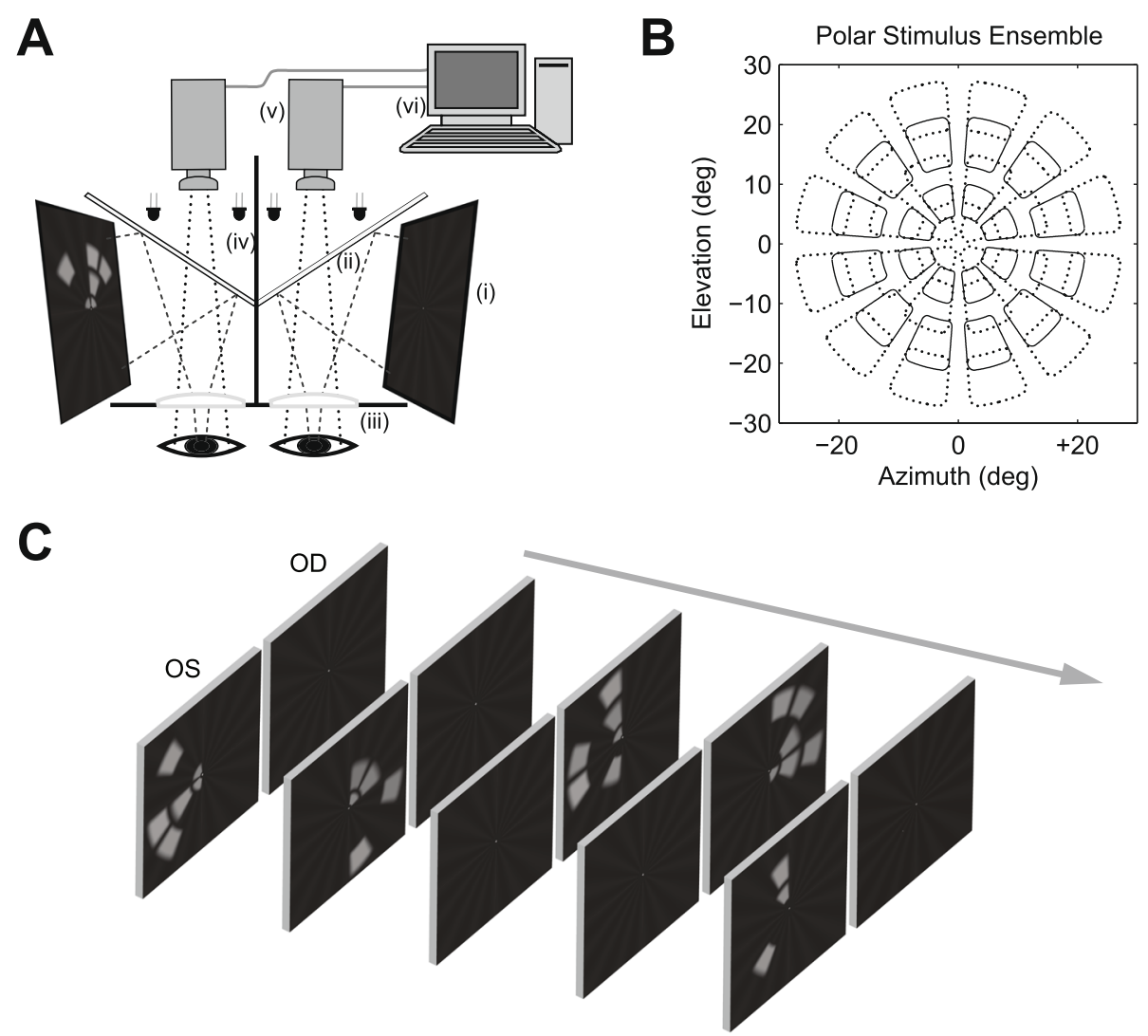

Fig. 2 Objective measurement of the visual fields. a Schematic of the objectiveFIELD Analyser ${ }^{\oplus}$ (OFA). Stimuli are presented independently on two liquid-crystal displays (i). The images are reflected by two dichroic mirrors (ii) allowing infrared light to pass while reflecting shorter wavelengths. Plano-convex lenses set the viewing distance to optical infinity (iii). Each eye views only one monitor, the images being fused by the subject into a cyclopean view. Infrared illumination of the eyes is provided by infrared light-emitting diodes (iv) which allows monitoring of each pupil by infrared video cameras (v). Pupil diameters are extracted in real-time and recorded by a computer (vi). b The 44 stimulus regions/ eye are arranged in a dartboard-like polar layout extending out to $30^{\circ}$ from fixation. Stimuli are pseudo-randomly presented to each hemifield of each eye in a consecutive series. c shows the randomised volleys of independent dichoptic stimuli across a series of video frames of the test sequence. Along with a fixation cross a faint stationary background starburst pattern assists the subjects to fuse the images

background illumination of $10 \mathrm{~cd} / \mathrm{m}^{2}$ adapted rod photoreceptor responses. The display included a small central (binocular) red fixation cross. Trial lenses were provided but to provide tolerance to mis-refraction the stimuli contained no spatial frequencies above 2 cycles/degree.

\section{Data analysis}

Analysis was conducted using MATLAB software (MathWorks, Natick, MA). Response waveforms - for both direct and consensual responses - from each region of the visual field were obtained and fitted to a log-normal function as follows:

$$
\mathrm{v}(\mathrm{t})=\mathrm{A} \exp \left(-\left[\operatorname{In}\left(\mathrm{t} / \mathrm{t}_{\mathrm{p}}\right)\right]^{2} / 2 \sigma^{2}\right)
$$

where $v(t)$ is the response waveform, A is the peak amplitude, $t$ is the time at which each estimation is made, $t_{p}$ is the time to peak, and $\sigma$ is the width of the response [15, 29].

This allowed the characterization of the responses according to standardized amplitude (AmpStd) and time-

Table 1 Stimulus characteristics of the blue and yellow stimulus protocols

\begin{tabular}{llllll}
\hline $\begin{array}{l}\text { Stimulus } \\
\text { protocol }\end{array}$ & $\begin{array}{l}\text { CIE } x, y \text { colour } \\
\text { coordinates }\end{array}$ & $\begin{array}{l}\text { Maximum luminance }(\mathbf{c d} / \\
\left.\mathbf{m}^{\mathbf{2}}\right)\end{array}$ & $\begin{array}{l}\text { Mean per-region } \\
\text { interval } \\
(\mathbf{s})\end{array}$ & $\begin{array}{l}\text { Per-region } \\
\text { duration } \\
(\mathbf{m s})\end{array}$ & $\begin{array}{l}\mathbf{N} \text { repeats per } \\
\text { region }\end{array}$ \\
\hline Blue (BP) & $0.145,0.113$ & 75 & 8 & 1000 & 45 \\
Yellow (YP) & $0.377,0.464$ & 150 & 4 & 33 & 90 \\
\hline
\end{tabular}




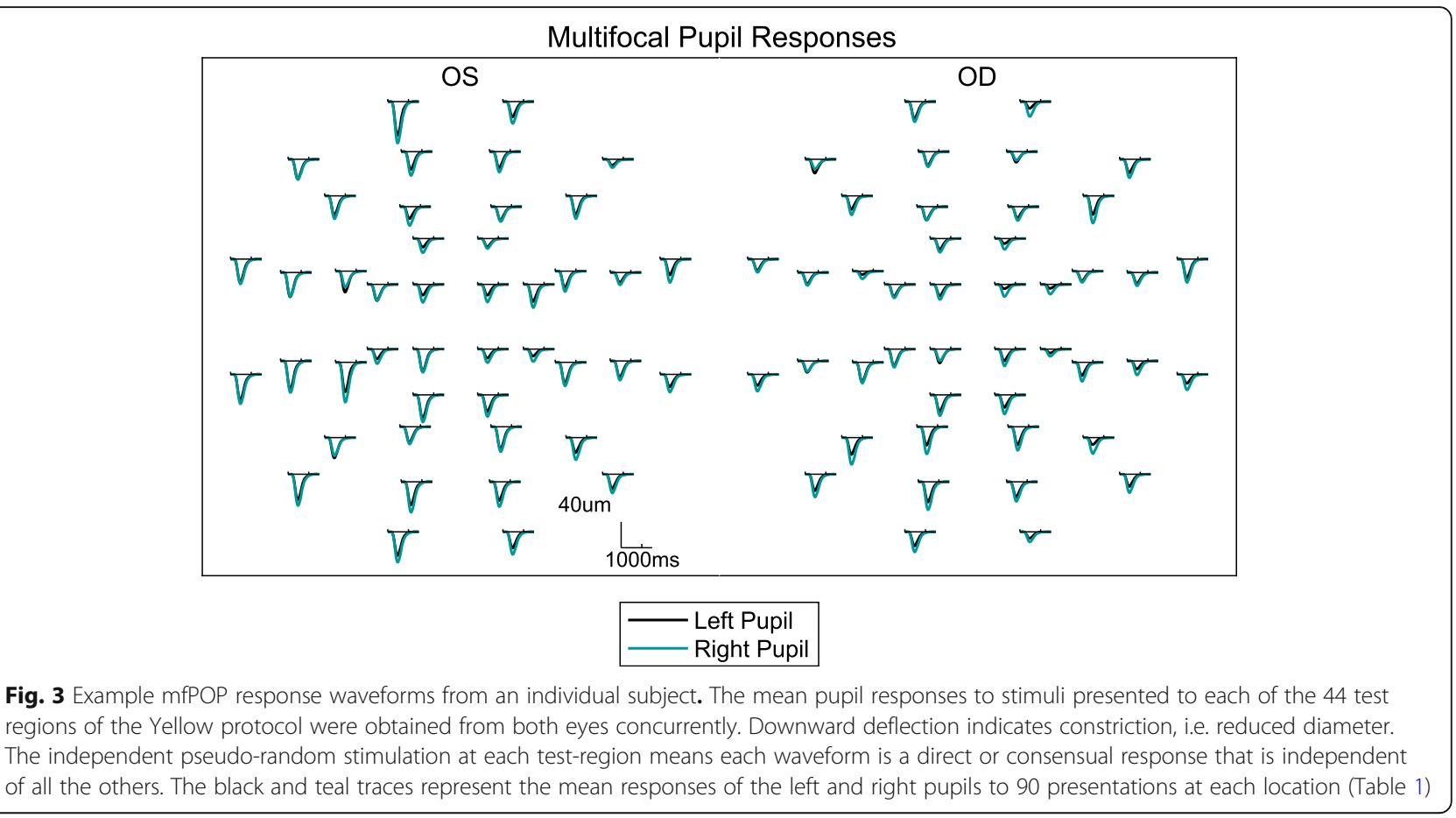

to-peak. AmpStd represents any change in pupil size corrected to the mean diameter of the population rather than using absolute pupil size and was expressed in decibels $(\mathrm{dB})$. It was derived from constriction amplitude as follows: AmpStd $=$ constriction amplitude $(\mu \mathrm{m}) \times 3500 / c$. Where $c$ is the mean pupil diameter based on the value of a line fitted to the entire $360 \mathrm{~s}$ of pupil diameter data recorded during each test, and $3500 \mu \mathrm{m}$ is the nominal population mean. AmpStd was used to overcome intersubject variation in mean pupil diameter and also improved tolerance to non-circular pupils, ansocoria, age and some drugs [31]. The higher the AmpStd, the larger the magnitude of pupillary constriction.

Student's t-test and Fisher's exact test were used to compare baseline characteristics. Odds ratios, McNemar's and Cochran's q tests, and one-way betweensubjects ANOVAs were conducted to compare the number of subjects developing migraine after each protocol - the primary outcome - and the association between mfPOP measures and other migraine parameters. Multivariate linear models were used to assess the independent effects of migraine parameters on the pupillary response.

The percentage area under the curve (AUC) of the receiver operating characteristic (ROC) plot was used as a measure of the power of mfPOP to predict migraine diagnosis i.e. it quantified the overall ability of mfPOP to discriminate between individuals with and without migraine. ROC plots were constructed for both AmpStd and time-to-peak in both protocols, using either the single worst region in each visual field (i.e. the one most deviating from normal) or the mean of the five worst regions, looking at either single eyes or at the asymmetry between anatomically-equivalent regions of the two eyes [15].

\section{Results}

Forty migraine patients were screened and 39 enrolled. Thirty-eight subjects completed testing with both mfPOP protocols. Two subjects were excluded because they developed a migraine within the $24 \mathrm{~h}$ prior to testing, one patient withdrew after the first test, and four subjects did not return completed diaries. In all, 32 sets of completed migraine diaries were returned and analyzed. In addition, 24 age- and sex-matched controls were studied (Table 2). Median acuity was $6 / 6$, one migraine subject was $6 / 12 \mathrm{OU}$. There was no significant difference in acuity between the control and migraine subjects.

\section{Effects of mfPOP testing on migraine}

Only one patient had difficulty completing the BP, reporting the occurrence of an aura at the end of the test. Otherwise, all patients reported no discomfort during testing apart from mild tearing due to insufficient blinking. The effects of testing on other migraine parameters are summarized in Table 3, which shows the same number of patients - four subjects (12.5\%) - developing a migraine attack in the first day after testing with either BP or YP. The difference was not significant (odds ratio 
Table 2 Subject characteristics

\begin{tabular}{lll}
\hline & $\begin{array}{l}\text { Subjects } \\
(\boldsymbol{n}=\mathbf{3 8})\end{array}$ & $\begin{array}{l}\text { Controls } \\
(\boldsymbol{n}=\mathbf{2 4})\end{array}$ \\
\hline Age (mean \pm SD) & $42.0 \pm 16.5$ & $39.2 \pm 15.2$ \\
Male: Female & $1: 1.8$ & $1: 1.5$ \\
Migraine type after BP or YP & - \\
•With aura & $26(72 \%)$ & \\
•Without aura & $15(42 \%)$ & \\
Mean age of onset (years) $( \pm$ SD) & $17.8 \pm 9.11$ & - \\
Mean disease duration (years) $( \pm$ SD) & $24.3 \pm 16.7$ & - \\
Treatment & & - \\
•Preventative & $8(22 \%)$ & \\
•During attacks & & \\
$\quad$ Over the counter & $25(69 \%)$ & \\
$\quad$ Triptans & $14(39 \%)$ & \\
$\quad$ Opioids & $13(36 \%)$ & \\
$\quad$ Ergot & $2(6 \%)$ & - \\
Mean attacks per month $( \pm$ SD) & $2.62 \pm 5.17$ & - \\
Mean headache duration (hours) $( \pm$ SD) & $11.75 \pm 16.5$ & - \\
Trigger & & - \\
•Light & $15(41 \%)$ & \\
•Other & $32(88 \%)$ & \\
Photophobia & $34(94 \%)$ & - \\
\hline
\end{tabular}

1.0; $95 \%$ confidence interval $0.2-4.4, p=0.48$ ). The results remained non-significant for both protocols over the first $72 \mathrm{~h}$, with 11 subjects (34.4\%) developing migraine after the BP and 13 subjects (41\%) after the YP (odds ratio 0.8 ; $95 \%$ confidence interval $0.3-2.1, p=$ 0.68). This period of $72 \mathrm{~h}$ was examined based on evidence that it may take up to $48 \mathrm{~h}$ following a trigger for a migraine to occur [39]. Although migraines occurred after testing the number of migraine days/week was not significantly increased relative to pre-testing: $1.4 \pm 1.6$ pre-testing (mean $\pm \mathrm{SD}$ ), $1.3 \pm 1.4$ post-BP, and $1.3 \pm 1.2$ post-YP ( $p=0.96$ for both). Other migraine parameters including attack severity, attack duration, and percentage of patients taking medication before and after each test were also not significantly different.

\section{Changes in mean mfPOP response characteristics}

Results for the mean (across all regions, eyes and pupils) AmpStd, time-to-peak and pupil diameter are summarized in Table 4. The pupil diameters were not different between control subjects and migraineurs, but the diameters for yellow stimuli were significantly larger than for blue $(\mathrm{t}=8.6, p<0.001)$. Being the means across regions etc. the results for AmpStd and time-to-peak mimic what might be obtained from a single large Ganzfield stimulus as is used in some pupil studies. No significant differences were found between patients and controls for either BP or YP.

In the absence of clear effects on the whole visual field, we next used a linear model to investigate localised defects. To minimise the effects of multiple comparisons we sorted the 44 regions in order of deviation from normal performance and then took the means of the 22/44 worst-performing regions as inputs to the linear model. This provided an assessment that made no a priori assumptions about any particular arrangement of localised visual field defects.

We fitted a factor for each of 3 increasingly long periods in which migraine occurred before testing, gender, age, medications, and other headaches. Of the various drugs, only triptans showed a significant effect. Overall, the closer a migraine attack occurred prior to the time of testing, the more negative the effect upon AmpStd, i.e. there was reduced sensitivity (Table 5). The greatest reduction was seen if the attack occurred within the week prior to testing, followed by 2 weeks and, lastly, 2 months before testing. The use of triptans was associated with a significant increase in AmpStd in both YP and BP $(0.45 \pm 0.09 \mathrm{~dB}$, and $0.48 \pm 0.07 \mathrm{~dB}$, respectively, $p<0.001)$. We fitted the identical model for times-topeak. In that model Triptan use was not significant, and other headaches and being female were less significant. The main effects of interest were that a migraine within the previous 2 weeks was associated with faster

Table 3 Effects of mfPOP stimulation on migraine headache severity parameters

\begin{tabular}{|c|c|c|c|c|}
\hline Parameter & Pre-testing & Post-BP & Post-YP & $P$-value \\
\hline Patients experiencing migraine in the 1st day post testing, no. (\%) & - & $4(12.5 \%)$ & $4(12.5 \%)$ & $0.48^{\mathrm{a}}$ \\
\hline Patients experiencing migraine within 3 days post testing, no. (\%) & - & $11(34.4 \%)$ & $13(41 \%)$ & $0.68^{\mathrm{a}}$ \\
\hline Migraine days/week (mean \pm SD) & $1.4 \pm 1.6$ & $1.3 \pm 1.4$ & $1.3 \pm 1.2$ & $0.96^{\mathrm{b}}$ \\
\hline$\%$ of patients experiencing 'quite bad' to 'very bad' migraine & $34 \%$ & $25 \%$ & $43 \%$ & $0.10^{c}$ \\
\hline Mean attack duration (hours) & 1.41 & 1.09 & 1.07 & $0.71^{b}$ \\
\hline$\%$ of patients taking medication & $50 \%$ & $50 \%$ & $53 \%$ & $0.93^{c}$ \\
\hline
\end{tabular}


Table 4 Mean BP and YP response characteristics of controls and migraineurs

\begin{tabular}{llll}
\hline & & $\begin{array}{l}\text { Control } \\
\text { (mean } \pm \text { SD) }\end{array}$ & $\begin{array}{l}\text { Migraine } \\
\text { (mean } \pm \text { SD) }\end{array}$ \\
\hline Blue protocol & AmpStd (dB) & $12.1 \pm 2.55$ & $11.8 \pm 2.49$ \\
& Time-to-peak (ms) & $594.9 \pm 53.3$ & $604.5 \pm 46.8$ \\
& Pupil Diameter (mm) & $2.68 \pm 0.29$ & $2.77 \pm 0.36$ \\
Yellow protocol & AmpStd (dB) & $12.3 \pm 1.77$ & $11.9 \pm 1.90$ \\
& Time-to-peak (ms) & $493.5 \pm 25.0$ & $495.2 \pm 21.9$ \\
& Pupil Diameter (mm) & $3.41 \pm 0.51$ & $3.51 \pm 0.74$ \\
\hline
\end{tabular}

responses: BP $21.9 \pm 3.50 \mathrm{~ms}$, and YP $29.7 \pm 1.77 \mathrm{~ms}(p<$ 0.001 for both). Both more recent and more distant migraines were associated with smaller changes in delay. There was no predictive association with a migraine that was about to occur in the week following testing, i.e. no changes in the pupillary response were seen before a migraine attack that was about to happen. There was no association with disease duration, age of onset, triggers or photosensitivity.

\section{Visual field defects detected by the mfPOP}

For each eye we derived deviation from normal performance, akin to the total deviations of a standard perimeter. The deviations were converted to Z-scores. Especially for $\mathrm{BP}$, the observed deviations from normal appeared to be fairly random, with no obvious clinically-relevant features such as homonymous defects. To look for any consistent patterns we computed the means of the Z-scores across pupils and eyes and then converted those to $p$ values at $p=0.05,0.02,0.01$ and 0.005 as is common for perimeters. Before averaging the polar mfPOP results were converted to a 24-2 pattern in order to facilitate comparison with standard perimetry. Details of the procedure have been provided elsewhere [40]. Given the results of the linear model, the averages for patients with different times since their last migraine were computed.
For BP, no defect survived the averaging, i.e. there was no consistent defect in any part of the visual field data following a pre-test migraine. For YP, however, patients with a migraine 1 to 2 weeks before testing demonstrated consistent inferotemporal defects. Figure 4 shows the average YP results for patients with a migraine longer ago than 60 days (Fig. 4a) and for those with a migraine within the last week (Fig. 4b). For subjects with migraines within the last 14 days, the 4 most significant regions of Fig. $4 \mathrm{~b}$ were at the $5 \%$ level.

The power of mfPOP to predict the diagnosis of migraine We next investigated the diagnostic power of BP and YP by examining the percentage area under the curve (\%AUC) of the receiver operating characteristic (ROC) curve when comparing control eyes to patient eyes. To compare data based on the entire visual fields of every eye, we collapsed the direct and consensual responses of every region using weightings based on the signal-tonoise ratios of each region [15]. Table 6 shows the results comparing the 24 subjects (48 eyes) who had had a migraine within 2 weeks prior to testing. Individual \%AUCs were then derived for several additional measures, specifically: the single worst performing (relative to normal) region in each visual field, the means of the worst 2 regions, the worst 3 , the worst 4 , etc. This allowed assessment of diagnostic performance as a function of the number of regions considered. We also derived ROCs for individual regional values for each eye as well as the difference between the mirror-image regions of the two eyes (generating 44 local 'asymmetry' measures/subject). For these ROCs, the largest \%AUC was for the single worst region of each field in the 'asymmetry' assessment of YP $(77.5 \% \pm 5.13 \%)$. AUC values for YP asymmetry also remained relatively high for the means of the worst 5 regions in each field $(76.7 \% \pm 4.93)$. This indicated that YP fields contained a larger number of diagnostically meaningful regions than BP fields. For

Table 5 Independent effects of headache on AmpStd in migraine subjects from a linear model

\begin{tabular}{|c|c|c|c|c|}
\hline & \multicolumn{2}{|l|}{ Blue protocol } & \multicolumn{2}{|c|}{ Yellow protocol } \\
\hline & $\mathrm{dB} \pm \mathrm{SE}$ & $P$ value & $\mathrm{dB} \pm \mathrm{SE}$ & $P$ value \\
\hline$\overline{\text { (Reference) }^{\mathrm{a}}}$ & $(-1.04 \pm 0.10)$ & - & $(-0.92 \pm 0.08)$ & - \\
\hline Migraine within last 60 days $(n=28)$ & $-0.45 \pm 0.16$ & 0.004 & $-0.08 \pm 0.12$ & 0.994 \\
\hline Migraine within last 2 weeks $(n=24)$ & $-0.64 \pm 0.14$ & $<0.001$ & $-0.65 \pm 0.14$ & $<0.001$ \\
\hline Migraine within last week $(n=19)$ & $-0.80 \pm 0.10$ & $<0.001$ & $-0.57 \pm 0.08$ & $<0.001$ \\
\hline Triptan use $(n=13)$ & $0.45 \pm 0.10$ & 0.006 & $0.49 \pm 0.07$ & $<0.001$ \\
\hline Other headaches $(n=2)$ & $2.39 \pm 0.25$ & $<0.001$ & $-1.16 \pm 0.14$ & $<0.001$ \\
\hline Female gender $(n=22)$ & $0.06 \pm 0.10$ & 1.000 & $0.41 \pm 0.08$ & $<0.001$ \\
\hline Age (dB per decade) $)^{b}$ & $-0.28 \pm 0.03$ & $<0.001$ & $-0.14 \pm 0.02$ & $<0.001$ \\
\hline
\end{tabular}

$P$-values are Bonferroni corrected

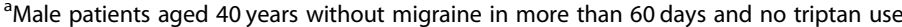

${ }^{\mathrm{b}} \mathrm{Age}$ was in decades relative to the median age of 40 years 


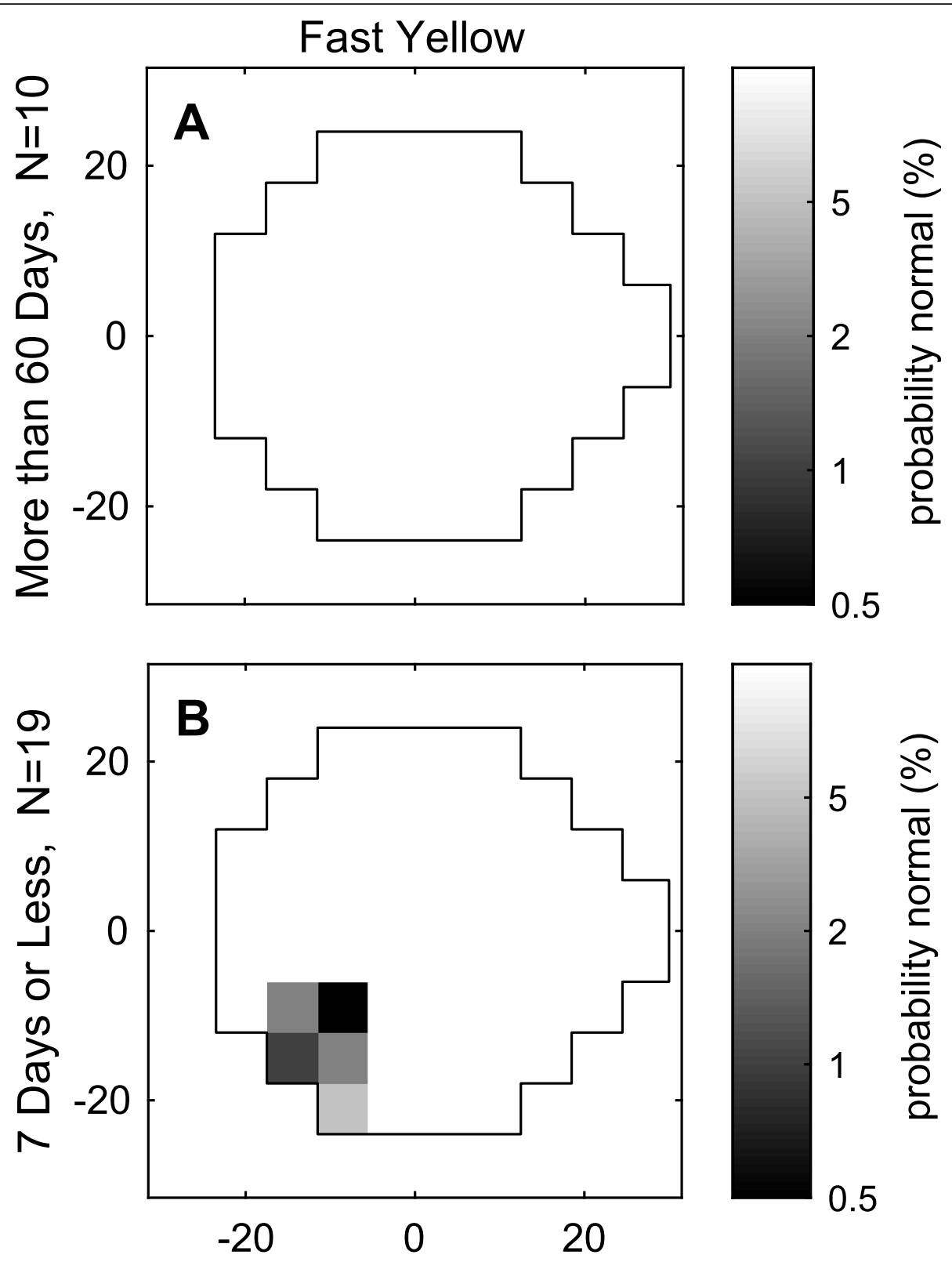

Fig. 4 Average visual field defects for yellow protocol for a last migraine longer ago than 60 days before testing, and $\mathbf{b}$ last migraine within 7 days or less. Before averaging across data for eyes and pupils the $z$-scores were converted to a 24-2 format to make the results more comparable to standard perimetry. Data from right eyes were flipped to resemble left eyes so that corresponding parts of the fields could be averaged. Hence, while the data from 10 and 19 subjects were used for $a$ and b, respectively, the actual number of fields averaged in each figure was 40 and 76 as this included fields for both eyes and both pupils. Despite 76 fields being averaged b shows a consistent inferior-temporal scotoma. The blue protocol data showed no consistent scotomas

subjects whose migraine had occurred within 14 days earlier the \%AUCs ranged from 52.8 to $65.4 \%$.

\section{Discussion}

This study's three aims were to determine (i), whether $\mathrm{BP}$ was more likely than YP to exacerbate migraine, (ii) to determine whether there were any abnormalities of pupillary function following an attack of migraine, and (iii) to determine the power of mfPOP to distinguish patients with migraine from normal controls.

In relation to the first aim, there was no detectable increase in the incidence or severity of migraine headaches when using the blue stimulus, which had been specifically designed to stimulate the melanopsin-containing ipRGCs [14]. Regarding the second aim, at a global level the average sensitivity and times-to-peak of migraine patients did not differ from those of controls (Table 4). 
Table $6 \%$ AUCS \pm SE for blue and yellow protocols comparing the 24 normal controls and the 24 patients experiencing migraine within 14 or fewer days before mfPOP testing

\begin{tabular}{lll}
\hline & Blue protocol (\%) & Yellow protocol (\%) \\
\hline AmpStd & & $66.6 \pm 5.65$ \\
. Worst region of $\mathbf{4 4}$ & $63.6 \pm 5.97$ & $65.2 \pm 5.74$ \\
. Mean of worst $\mathbf{5}$ regions & $59.8 \pm 6.09$ & $77.5 \pm 5.13$ \\
Asymmetry between eyes & & $76.7 \pm 4.93$ \\
. Worst region of $\mathbf{4 4}$ & $76.8 \pm 5.05$ & \\
. Mean of worst $\mathbf{5}$ regions & $70.5 \pm 5.42$ & \\
\hline
\end{tabular}

This finding is consistent with that of Cambron et al. [17], who examined patients during both ictal and interictal phases using standard pupillometry. However, looking at individual regions, we found significant reductions in regional sensitivities associated with a migraine attack occurring within 2 weeks before testing (Tables 5, 6 and Fig. 4). Regarding the third aim, the diagnostic power of those regional changes was modest but, at least for YP there were 5 or more regions of asymmetry between eyes per field that were quite diagnostic (Table 6) and about 5 inferotemporal regions of the YP fields showed consistent defects (Fig. 4).

Triptan use was associated with increases in perregion sensitivities. Changes in per-region times-to-peak were less consistent but there was possibly an increase in speed of response associated with a migraine attack occurring within the 2 weeks before testing. Given that time-to-peak was somewhat affected suggests that the triptans could have had an effect on cortical hyperexcitability [41] but this clearly requires further investigation.

As pointed out by $[35,36]$ at the background and stimulus used here pupil responses to blue stimuli are substantially driven by melanopsin, event to stimuli as short as 1 to $2 \mathrm{~s}$. The times-to-peak for the BP were 100 ms longer than for YP (Table 4). This is in line with some melanopsin responses. We cannot rule out some rod intrusion however $[35,36]$. The human melanopsindriven response of the pupils can persist to over $2 \mathrm{~Hz}$ suggesting minimum latencies around $500 \mathrm{~ms}$ [42]. Responses of ipRGCs in dopaminergic amacrine cells can occur within $200 \mathrm{~ms}$ of light onset [7], much slower than rods or cones. The different types of ipRGCs differ in their physiology with M1 cells having the lowest thresholds and the fastest peak latencies at about $1 \mathrm{~s}$ [8].

It has recently been suggested that ipRGCs may be hyperactive in migraine [43], at least in persons with photophobia [5]. This might cause smaller pupils in such patients. Note that smaller pupils do not affect the relative measure AmpStd used here. The lack of any difference in pupil diameter between migraineurs and controls (Table 4) would indicate this was not happening here. That may be because a minority of our patients $(15 / 38)$ reported any photosensitivity and no photophobia post-test. Absolute pupil diameter was significantly smaller for the blue stimuli (Table $4, \mathrm{t}=8.6$, $p<0.0001$ ), suggesting that the blue stimuli were driving melanopsin. It is possible however that the dynamic driving of ipRGC was not as great as other studies where photophobia was enhanced by strongly activating melanopsin [5, 43].

\section{Distribution of sensitivity and retinal changes}

Abnormalities on flicker perimetry have been reported to persist for 7 days following an episode of migraine [19]. Of note, two of the migraine-induced flicker-fields in the literature demonstrated an inferotemporal defect $[19,20]$, similar to our findings (Fig. 4). These local visual field changes are unlikely to be due to generalized autonomic dysfunction of the pupil as this would be most likely to affect all regions of the visual field. Interestingly, the retinal nerve fibre layer (RNFL) of migraineurs can show thinning: a meta-analysis of six studies indicated that the thinning was most obvious superiorly, nasal retina being marginally more affected than temporal retina [44]. Superonasal retinal defects would correspond to the inferotemporal visual field defects found in this study. However, while an explanation at retinal level is possible, there may also be an effect at the cerebral cortex: pattern VEPs have been found to be abnormal in migraine $[45,46]$ while simultaneously-recorded pattern ERGs have not [45]. Interestingly, interictally recorded VEPs indicated hypersensitivity [45]. This would appear to agree with the recent work on ipRGCs and pupil responses during the interictal period [5]. Here we showed decreased sensitivity within 1 to 2 weeks after migraine attack for yellow stimuli, and for up to 60 days for blue (Table 5). Thus, migraine may represent an abnormally large fluctuation of ipRGC sensitivity pre- and post-migraine.

Perimetric defects reported for migraine tend to be sporadically distributed (like the BP fields here which averaged to zero), meaning that the Pattern Standard Deviation is often a better indicator of abnormality than the overall Mean Defect [19, 21, 22]. A similar effect has been observed in perimetric studies of concussion [47]. It is possible that both retinal and cortical effects 
contribute to the observed abnormalities, with YP being more sensitive to retinal abnormalities than BP [14]. Consistent with this is the observation that, in migraine, sensitivity to peripherally-presented contrast is more affected than contrast generated centrally [48]. A related finding is that alteration of peripheral contrast sensitivity has been shown to precede migraine onset by 1 to 2 days [49] though the stimuli of that study could not provide any information relating to any dominant contribution by one or more quadrants.

\section{mfPOP stimuli and visual distress}

With the emergence of new modalities to treat migraine such as pharmacological manipulation of melanopsin [50] or blocking of blue wavelengths using tinted lenses [51], it is important to understand the effects of ipRGC stimulation on migraine pathophysiology. This study is the first to use a stimulus specifically designed to target the melanopsin-containing ipRGCs in order to look for an effect on migraine occurrence [14]. We compared this stimulus (BP) with an established stimulus designed to stimulate transient luminance pathways (YP). It is worth noting, however, that the $\mathrm{BP}$ stimulus design tended to reduce characteristics that could potentially cause discomfort in migraine subjects. For example, the stimuli were delivered randomly to different locations in the visual field rather than synchronously across the whole field, so each stimulus activated much less than $10 \%$ of the visual cortex [52]. Similarly, the stimuli did not contain stripes or checks [53], had smooth edges like sine-wave gratings, and contained no spatial frequencies above $2.0 \mathrm{cycles} / \mathrm{deg}$. Thus, blue color and perpresentation stimulus-duration $(1000 \mathrm{~ms})$ were the only major characteristics that could have contributed to any effect that the BP stimuli might have had on triggering a migraine attack. We have used mfPOP stimuli that employed larger rectangular patterned stimuli [31], if these were blue and presented for longer durations it is possible that they could be a more effective probe of the contribution of ipRGCs to migraine.

\section{Conclusions}

This study has demonstrated that stimulation of melanospin-containing ipRGCs did not alter migraine parameters. Localized field defects were detected if testing was carried out within 2 weeks after an attack. It also suggested that mfPOP may prove to be an important tool to study visual pathophysiology in migraineurs in the future.

\section{Abbreviations}

AUC: Area under the curve; CSD: Cortical spreading depression; ipRGCs: Intrinsically-photosensitive retinal gangion cells; mfPOP: Mulifocal pupillographic objective perimetry; mfVEP: Multifocal visual evoked potential;
OFA: ObjectiveFIELD analyser; OTC: Over-the-counter medications; ROC: Receiver operating characteristic; RNFL: Retinal nerve fibre layer

\section{Code availability}

available on request

\section{Authors' contributions}

ENA designed the study, collected most of the data, and analyzed the data. CFC supervised Ali and assisted with testing, analysis and writing. CJL supervised ENA, provided clinical oversight, and assisted with writing. MK did ophthalmic testing and assisted with writing. TM supervised ENA, assisted with analysis and writing. The author(s) read and approved the final manuscript.

\section{Funding}

This work was supported by the Australian Research Council through the ARC Centre of Excellence in Vision Science (CE0561903). The funding body had no part in the design of the study, data collection, analysis, or interpretation of data, or in writing the manuscript.

\section{Availability of data and materials}

The datasets used and/or analysed during the current study available from the corresponding author on reasonable request.

\section{Declarations}

Ethics approval and consent to participate

Informed, written consent was obtained from all subjects. The study conformed to the Declaration of Helsinki guidelines and was approved by both the Human Research Ethics Committee of the Australian National University (2012/278) and the Australian Capital Territory (ACT) Health Human Research Ethics Committee (ETH.3.12.064). ACT Health manages the Hospital system for the Canberra region.

\section{Consent for publication}

Not Applicable

\section{Competing interests}

TM and CFC have assigned patents to Konan Medical USA for aspects of the mfPOP technology, and as such they may earn royalty income from that IP. ENA, CJL and MK have no conflicts.

\section{Author details}

${ }^{1}$ Eccles Institute of Neuroscience, the John Curtin School of Medical Research, Australian National University, Acton, ACT, Australia. ${ }^{2}$ Department of Neuroscience, King Fahad Specialist Hospital, Dammam, Saudi Arabia.

${ }^{3}$ Australian National University Medical School, Acton, ACT, Australia.

${ }^{4}$ Department of Neurology, The Canberra Hospital, Canberra, ACT, Australia.

Received: 29 November 2020 Accepted: 14 May 2021

Published online: 26 May 2021

\section{References}

1. Grafstein B. Mechanism of spreading cortical depression. J Neurophysiol. 1956;19(2):154-71. https://doi.org/10.1152/jn.1956.19.2.154.

2. Martin PR. Behavioral management of migraine headache triggers: learning to cope with triggers. Curr Pain Headache Rep. 2010;14(3):221-7. https://doi. org/10.1007/s11916-010-0112-z.

3. Noseda R, Kainz V, Jakubowski M, Gooley JJ, Saper CB, Digre K, et al. A neural mechanism for exacerbation of headache by light. Nat Neurosci. 2010;13(2):239-45. https://doi.org/10.1038/nn.2475.

4. Noseda R, Copenhagen D, Burstein R. Current understanding of photophobia, visual networks and headaches. Cephalalgia. 2019;39(3):162334. https://doi.org/10.1177/0333102418784750.

5. Zele AJ, Dey A, Adhikari P, Feigl B. Melanopsin hypersensitivity dominates interictal photophobia in migraine. Cephalalgia. 2021;41(2):217-26 https:// journals.sagepub.com/doi/10.1177/0333102420963850.

6. Dacey DM, Liao HW, Peterson BB, Robinson FR, Smith VC, Pokorny J, et al. Melanopsin-expressing ganglion cells in primate retina signal colour and irradiance and project to the LGN. Nature. 2005;433(7027):749-54. https:// doi.org/10.1038/nature03387. 
7. Prigge $C L$, Yeh PT, Liou NF, Lee CC, You SF, Liu LL, et al. M1 ipRGCs influence visual function through retrograde signaling in the retina. J Neurosci. 2016;36(27):7184-97. https://doi.org/10.1523/JNEUROSCI.3500-15.2 016.

8. Zhao X, Stafford BK, Godin AL, King WM, Wong KY. Photoresponse diversity among the five types of intrinsically photosensitive retinal ganglion cells. J Physiol. 2014;592(7):1619-36. https://doi.org/10.1113/jphysiol.2013.262782.

9. Kawasaki A, Kardon RH. Intrinsically photosensitive retinal ganglion cells. J Neuroophthalmol. 2007;27(3):195-204. https://doi.org/10.1097/WNO.0b013 e31814b1df9.

10. Park JC, Moura AL, Raza AS, Rhee DW, Kardon RH, Hood DC. Toward a clinical protocol for assessing rod, cone, and melanopsin contributions to the human pupil response. Invest Ophthalmol Vis Sci. 2011;52(9):6624-35. https://doi.org/10.1167/iovs.11-7586.

11. Adhikari P, Feigl B, Zele AJ. Rhodopsin and melanopsin contributions to the early redilation phase of the post-illumination pupil response (PIPR). PLOS One. 2016;11(8):e0161175. https://doi.org/10.1371/journal.pone.0161175.

12. Gracitelli CP, Duque-Chica GL, Moura AL, Nagy BV, de Melo GR, Roizenblatt $M$, et al. A positive association between intrinsically photosensitive retinal ganglion cells and retinal nerve fiber layer thinning in glaucoma. Invest Ophthalmol Vis Sci. 2014;55(12):7997-8005. https://doi.org/10.1167/iovs.14-1 5146.

13. Main A, Vlachonikolis I, Dowson A. The wavelength of light causing photophobia in migraine and tension-type headache between attacks. Headache. 2000;40(3):194-9. https://doi.org/10.1046/j.1526-4610.2000.00028. $\mathrm{x}$.

14. Carle CF, James AC, Kolic M, Essex RW, Maddess T. Blue multifocal pupillographic objective perimetry in glaucoma. Invest Ophthalmol Vis Sci. 2015;56(11):6394-403. https://doi.org/10.1167/iovs.14-16029.

15. Bell A, James AC, Kolic M, Essex RW, Maddess T. Dichoptic multifocal pupillography reveals afferent visual field defects in early type 2 diabetes. Invest Ophthalmol Vis Sci. 2010;51(1):602-8. https://doi.org/10.1167/iovs.09-3 659.

16. Carle CF, Maddess T, James AC. Contraction anisocoria: segregation, summation, and saturation in the pupillary pathway. Invest Ophthalmol Vis Sci. 2011:52(5):2365-71. https://doi.org/10.1167/iovs.10-6335.

17. Cambron M, Maertens H, Paemeleire K, Crevits L. Autonomic function in migraine patients: ictal and interictal pupillometry. Headache. 2013;54(4): 655-62. https://doi.org/10.1111/head.12139.

18. Barriga FJ, Lopez de Silanes C, Gili P, Pareja JA. Ciliary ganglioplegic migraine: migraine-related prolonged mydriasis. Cephalalgia. 2011;31(3):2915. https://doi.org/10.1177/0333102410381144.

19. McKendrick AM, Badcock DR. Decreased visual field sensitivity measured 1 day, then 1 week, after migraine. Invest Ophthalmol Vis Sci. 2004;45(4): 1061-70. https://doi.org/10.1167/iovs.03-0936.

20. McKendrick AM, Badcock DR. An analysis of the factors associated with visual field deficits measured with flickering stimuli in-between migraine. Cephalalgia. 2004;24(5):389-97. https://doi.org/10.1111/j.1468-2982.2004. 00682.x.

21. Yenice O, Temel A, Incili B, Tuncer N. Short-wavelength automated perimetry in patients with migraine. Graefes Arch Clin Exp Ophthalmol. 2006;244(5):589-95. https://doi.org/10.1007/s00417-005-0083-7.

22. Yener $\mathrm{AU}$, Korucu O. Visual field losses in patients with migraine without aura and tension-type headache. Neuro-Ophthalmol. 2017;41(2):59-67. https://doi.org/10.1080/01658107.2016.1251466.

23. Harle DE, Evans BJW. Frequency doubling technology perimetry and standard automated perimetry in migraine. Ophthalmic Physiol Opt. 2005; 25(3):233-9. https://doi.org/10.1111/j.1475-1313.2005.00285.x.

24. Sabeti F, Maddess T, Essex RW, James AC. Multifocal pupillography identifies ranibizumab-induced changes in retinal function for exudative age-related macular degeneration. Invest Ophthalmol Vis Sci. 2012;53(1):253-60. https:// doi.org/10.1167/iovs.11-8004

25. Ali E, Maddess T, James A, Voicu C, Lueck C. Pupillary response to sparse multifocal stimuli in multiple sclerosis patients. Mult Scler. 2014;20:854-61. https://doi.org/10.1177/1352458513512708.

26. Sabeti F, Carle CF, Jaros RK, Rohan EMF, J LC, Hughes D, et al. Objective perimetry in sporting-related mild traumatic brain injury. Ophthalmology. 2019;126(7):1053-5. https://doi.org/10.1016/j.ophtha.2019.01.026.

27. Sabeti F, James AC, Carle CF, Essex RW, Bell A, Maddess T. Comparing multifocal pupillographic objective perimetry (mfPOP) and multifocal visual evoked potentials (mfVEP) in retinal diseases. Sci Rep. 2017;7(1):45847. https://doi.org/10.1038/srep45847.

28. Rosli Y, Carle CF, Ho Y, James AC, Kolic M, Rohan EMF, et al. Retinotopic effects of visual attention revealed by dichoptic multifocal pupillography. Sci Report. 2018;8(1):2991. https://doi.org/10.1038/s41598-018-21196-1.

29. Carle CF, James AC, Maddess T. The pupillary response to color and luminance variant multifocal stimuli. Invest Ophthalmol Vis Sci. 2013;54(1): 467-75. https://doi.org/10.1167/iovs.12-10829.

30. IHS Headache Classification Committee. The international classification of headache disorders, 3rd edition. Cephalalgia. 2018;38(1):1-211. https://doi. org/10.1177/0333102417738202.

31. Maddess T, Bedford SM, Goh XL, James AC. Multifocal pupillographic visual field testing in glaucoma. Clin Exp Ophthalmol. 2009;37(7):678-86. https:// doi.org/10.1111/j.1442-9071.2009.02107.x.

32. Lawrence M, Friedman CDF, DeMets DL. Fundamentals of clinical trials. New york: Springer; 1998. ISBN 978-3-319-18539-2.

33. Urbaniak GC, Plous S. Research randomizer In., 4.0 edn. 2013. http://www.ra ndomizer.org/.

34. Jensen R, Tassorelli C, Rossi P, Allena M, Osipova V, Steiner T, et al. A basic diagnostic headache diary (BDHD) is well accepted and useful in the diagnosis of headache. a multicentre European and Latin American study. Cephalalgia. 2011;31(15):1549-60. https://doi.org/10.1177/03331 02411424212.

35. McDougal DH, Gamlin PD. The influence of intrinsically-photosensitive retinal ganglion cells on the spectral sensitivity and response dynamics of the human pupillary light reflex. Vis Res. 2010;50(1):72-87. https://doi.org/1 0.1016/j.visres.2009.10.012.

36. Herbst K, Sander B, Lund-Andersen H, Broendsted AE, Kessel L, Hansen MS, et al. Intrinsically photosensitive retinal ganglion cell function in relation to age: a pupillometric study in humans with special reference to the agerelated optic properties of the lens. BMC Ophthalmol. 2012;12(1):4. https:// doi.org/10.1186/1471-2415-12-4.

37. Sabeti F, Maddess T, Essex RW, Saikal A, James AC, Carle CF. Multifocal pupillography in early age-related macular degeneration. Optom Vis Sci. 2014;91(8):904-15. https://doi.org/10.1097/opx.0000000000000319.

38. Carle CF, James AC, Rosli Y, Maddess T. Localization of neuronal gain control in the pupillary response. Front Neurol. 2019;10(203):1-9. https://doi. org/10.3389/fneur.2019.00203.

39. Zagami A, Bahra A. Symptomatology of migraines without aura. In the headaches. 3rd ed. Philadelphia: Lippincott Williams \& Wilkins; 2006.

40. Ruseckaite R, Maddess T, James AC. Frequency doubling illusion VEPs and automated perimetry in multiple sclerosis. Doc Ophthalmol. 2006;113(1):29_ 41. https://doi.org/10.1007/s10633-006-9011-3.

41. Coppola G, Schoenen J. Cortical excitability in chronic migraine. Curr Pain Headache Rep. 2012;16(1):93-100. https://doi.org/10.1007/s11916-011-0231-1.

42. Spitschan M, Jain S, Brainard DH, Aguirre GK. Opponent melanopsin and Scone signals in the human pupillary light response. Proc Natl Acad Sci U S A. 2014;111(43):15568-72. https://doi.org/10.1073/pnas.1400942111.

43. McAdams H, Kaiser EA, Igdalova A, Haggerty EB, Cucchiara B, Brainard DH, et al. Selective amplification of ipRGC signals accounts for interictal photophobia in migraine. Proc Natl Acad Sci U S A. 2020;117(29):17320-9. https://doi.org/10.1073/pnas.2007402117.

44. Feng YF, Guo H, Huang JH, Yu JG, Yuan F. Retinal nerve fiber layer thickness changes in migraine: a meta-analysis of case-control studies. Curr Eye Res. 2016:41(6):814-22. https://doi.org/10.3109/02713683.2015.1056373.

45. Nguyen BN, McKendrick AM, Vingrys AJ. Simultaneous retinal and cortical visually evoked electrophysiological responses in between migraine attacks. Cephalalgia. 2012;32(12):896-907. https://doi.org/10.1177/0333102412453953.

46. Nguyen BN, McKendrick AM, Vingrys AJ. Abnormal inhibition-excitation imbalance in migraine. Cephalalgia. 2015;36(1):5-14. https://doi.org/10.1177/ 0333102415576725.

47. Walsh DV, Capo-Aponte JE, Jorgensen-Wagers K, Temme LA, Goodrich G, Sosa J, et al. Visual field dysfunctions in warfighters during different stages following blast and nonblast mTBI. Mil Med. 2015;180(2):178-85. https://doi. org/10.7205/MILMED-D-14-00230.

48. Battista J, Badcock DR, McKendrick AM. Migraine increases centre-surround suppression for drifting visual stimuli. PLoS One. 2011;6(4):e18211. https:// doi.org/10.1371/journal.pone.0018211.

49. McKendrick AM, Chan YM, Vingrys AJ, Turpin A, Badcock DR. Daily vision testing can expose the prodromal phase of migraine. Cephalalgia. 2018; 38(9):1575-84. https://doi.org/10.1177/0333102417741130. 
50. Jones KA, Hatori M, Mure LS, Bramley JR, Artymyshyn R, Hong SP, et al. Small-molecule antagonists of melanopsin-mediated phototransduction. Nat Chem Biol. 2013;9(10):630-5. https://doi.org/10.1038/nchembio.1333.

51. Good PA, Taylor RH, Mortimer MJ. The use of tinted glasses in childhood migraine. Headache. 1991;31(8):533-6. https://doi.org/10.1111/j.1526-4610.1 991.hed3108533.x.

52. Hay KM, Mortimer MJ, Barker DC, Debney LM, Good PA. 1044 women with migraine: the effect of environmental stimuli. Headache. 1994;34(3):166-8. https://doi.org/10.1111/j.1526-4610.1994.hed3403166.x.

53. Marcus DA, Soso MJ. Migraine and stripe-induced visual discomfort. Arch Neurol. 1989;46(10):1129-32. https://doi.org/10.1001/archneur.1989.005204 60125024

\section{Publisher's Note}

Springer Nature remains neutral with regard to jurisdictional claims in published maps and institutional affiliations.

Ready to submit your research? Choose BMC and benefit from:

- fast, convenient online submission

- thorough peer review by experienced researchers in your field

- rapid publication on acceptance

- support for research data, including large and complex data types

- gold Open Access which fosters wider collaboration and increased citations

- maximum visibility for your research: over $100 \mathrm{M}$ website views per year

At $B M C$, research is always in progress.

Learn more biomedcentral.com/submissions 